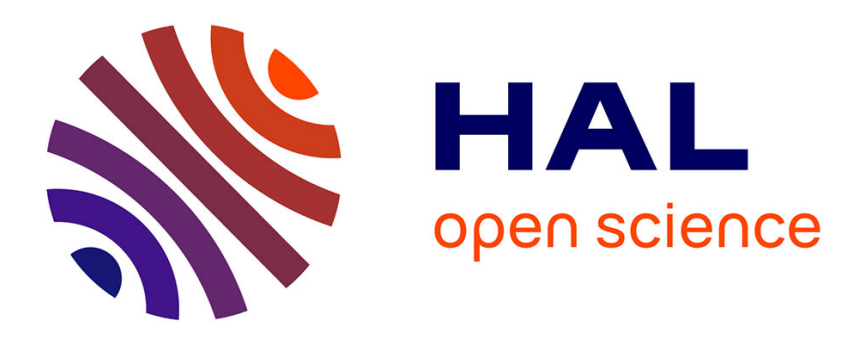

\title{
Stabilisation en fréquence et en intensité d'un laser monomode de puissance grâce au lamb dip magnétique
}

\author{
A. Le Floch, P. Frere
}

\section{To cite this version:}

A. Le Floch, P. Frere. Stabilisation en fréquence et en intensité d'un laser monomode de puissance grâce au lamb dip magnétique. Revue de Physique Appliquée, 1972, 7 (4), pp.409-412. 10.1051/rphysap:0197200704040900 . jpa-00243646

\section{HAL Id: jpa-00243646 https://hal.science/jpa-00243646}

Submitted on 1 Jan 1972

HAL is a multi-disciplinary open access archive for the deposit and dissemination of scientific research documents, whether they are published or not. The documents may come from teaching and research institutions in France or abroad, or from public or private research centers.
L'archive ouverte pluridisciplinaire HAL, est destinée au dépôt et à la diffusion de documents scientifiques de niveau recherche, publiés ou non, émanant des établissements d'enseignement et de recherche français ou étrangers, des laboratoires publics ou privés. 


\title{
STABILISATION EN FRÉQUENCE ET EN INTENSITÉ D'UN LASER MONOMODE DE PUISSANCE GRÂCE AU LAMB DIP MAGNÉTIQUE
}

\author{
A. LE FLOCH et P. FRERE \\ Laboratoire d'Electronique Quantique \\ Faculté des Sciences, 35, Rennes \\ (Reçu le 1er juin 1972, révisé le 28 août 1972)
}

\begin{abstract}
Résumé. - Une méthode de stabilisation en fréquence et en intensité d'un laser monomode de puissance est proposée. Elle n'utilise pas une cavité de référence stable mais une référence atomique basée sur le Lamb dip magnétique qui permet de fixer la fréquence grâce à une première boucle de contre-réaction agissant sur la cavité contenant le milieu actif. Une seconde boucle stabilise la cavité passive qui détermine la puissance de sortie.
\end{abstract}

\begin{abstract}
A frequency and power stabilization method for a high power monomode laser is proposed. It does not use a stable reference cavity but an atomic resonance, the magnetic Lamb dip, which provides a discriminant for a first feedback loop stabilizing the active cavity. A second feedback loop stabilizes the passive cavity which determines the output power.
\end{abstract}

I. Introduction. - La méthode usuelle pour obtenir un laser à gaz monomode consiste à réduire la longueur de la cavité pour que l'écart de fréquences entre deux modes axiaux successifs soit comparable à la largeur Doppler de la raie. Il en résulte malheureusement que la puissance de sortie du laser devient faible (par exemple de l'ordre de $0,1 \mathrm{~mW}$ pour la raie $6328 \AA$ du néon). Ceci est gênant pour certaines applications comme la spectroscopie Brillouin à haute résolution [1] où on cherche à avoir une fréquence stable et une intensité supérieure à celle donnée par un laser monomode à cavité courte.

Divers systèmes [2] ont été proposés pour obtenir un sélecteur de mode permettant de faire osciller un seul des modes d'un laser long, normalement multimode. L'interféromètre du type Fox-Smith utilisant trois miroirs a permis d'obtenir un laser monomode d'une puissance de $50 \mathrm{~mW}$ sur la raie $6328 \AA$. Cependant la plus grande complexité du résonateur entraîne des fluctuations importantes de la fréquence et de l'intensité du faisceau de sortie et nécessite pour beaucoup de manipulations un système de stabilisation. Les méthodes proposées jusqu'ici, pour l'interféromètre du type Fox-Smith, qui se compose de deux cavités Pérot-Fabry couplées, reposent sur la stabilité de la cavité passive. On transfère la stabilité d'une cavité passive, courte, réalisée en invar, à la cavité active par une boucle de contre-réaction [3], [4]. Cependant, toute dérive de la cavité de référence se répercute sur la fréquence du laser. Nous proposons ici une méthode utilisant une référence atomique pour fixer la fréquence d'oscillation du laser, le « Lamb dip magnétique » que nous avons mis en évidence expérimentalement antérieurement (5).

L'intensité de sortie est stabilisée par une seconde boucle agissant sur la cavité passive.

II. Laser à interféromètre de Fox-Smith. Effet d'un champ magnétique axial. - $-a)$ L'INTERFÉROMÈTRE DE Fox-SMITH. - La figure 1 rappelle le schéma du

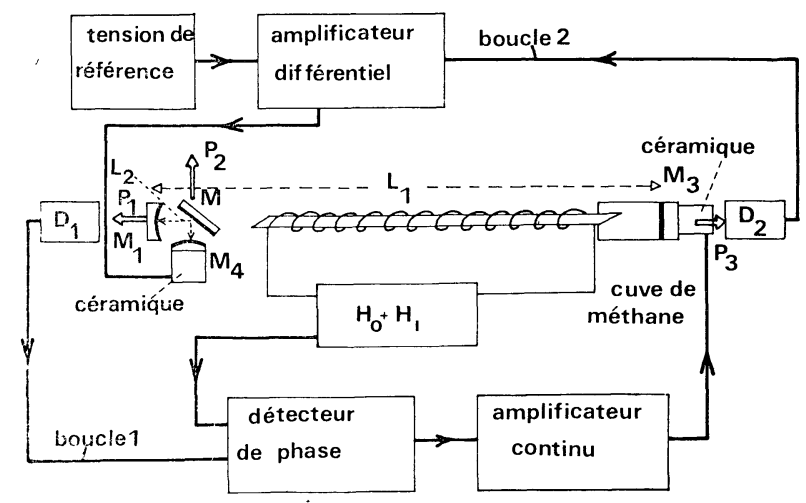

FIG. 1. - Montage expérimental.

résonateur utilisé. Le laser comporte deux cavités distinctes de longueur $L_{1}$ et $L_{2}$ qui sont couplées par le miroir commun, $M_{1}$. Il sera donc possible de corriger la longueur des deux cavités séparément, en agissant sur les miroirs $\mathbf{M}_{3}$ et $\mathbf{M}_{4}$. La cavité longue de longueur $L_{1}\left(L_{1}=180 \mathrm{~cm}\right)$, contient le milieu amplificateur et constitue la cavité active. La cavité courte de longueur $L_{2}\left(L_{2}=10 \mathrm{~cm}\right)$ est passive et 
permet de sélectionner un des modes de la cavité $L_{1}$. L'interféromètre de Fox-Smith constitué par $M_{1}, M_{2}$, $\mathbf{M}_{4}$ formant la cavité $L_{2}$ se comporte comme un miroir dont le pouvoir réflecteur en fonction de la fréquence est identique à la transmission d'un PérotFabry de même longueur. Le mode oscillant correspond à la coïncidence d'un mode de la cavité active $L_{1}$ et d'un mode de la cavité passive $L_{2}$ (Fig. 2). La fré-

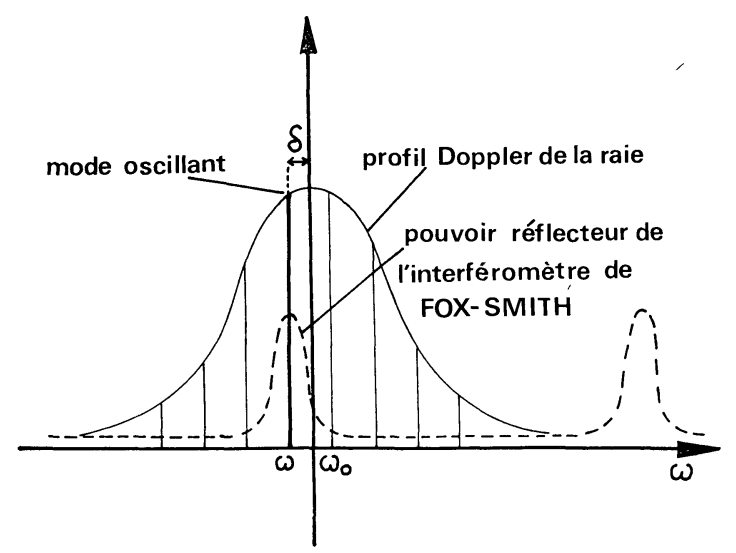

FIG. 2.

quence du mode est fixée par le bras actif et l'intensité de sortie par le bras passif qui fixe le pouvoir réflecteur du sélecteur de mode, de façon continue. Lorsque l'interféromètre a un pouvoir réflecteur maximum la puissance à l'intérieur du laser est maximale c'est-àdire que $P_{1}$ et $P_{3}$ sont maximales alors que la puissance utile $P_{2}$ est minimale. Une modulation de la longueur de la cavité $L_{2}$ conduit aux variations respectives de $P_{2}$ et $P_{3}$ représentées sur la figure 3. Les résultats sont analogues à ceux obtenus par Smith [3]. On remarque qu'aux points de fonctionnement donnant des puissances $P_{2}$ maximales correspondent des variations importantes de $P_{3}$ ce qui permettra de stabiliser l'intensité.

b) ACTION D'UN CHAMP MAGNÉTIQUe AXIAL. L'application d'un champ axial $\mathbf{H}$ décompose la raie $6328 \AA(J=1 \rightarrow J=2)$ en deux composantes Zeeman $\sigma^{+}$et $\sigma^{-}$(les facteurs de Landé des niveaux $3 s_{2}$ et $2 p_{4}$ sont en effet très voisins). L'étude de l'effet Zeeman conduit à étudier les variations de polarisation et d'amplitude du vecteur électrique oscillant, en fonction du champ appliqué.

En champ nul, la polarisation du vecteur champ électrique est pratiquement linéaire et imposée par les fenêtres de Brewster, qui représentent des anisotropies fortes. Dans le cas général, en présence d'un champ magnétique axial variable, on montre [6] que la polarisation d'un laser anisotrope accordé sur la fréquence centrale de la raie reste linéaire mais subit une rotation qui peut atteindre une valeur maximum située entre $45^{\circ}$ et $90^{\circ}$ en fonction de la valeur de l'anisotropie de la cavité. Au-delà d'une valeur critique $H_{c}$ du champ magnétique, on obtient une décomposition de la

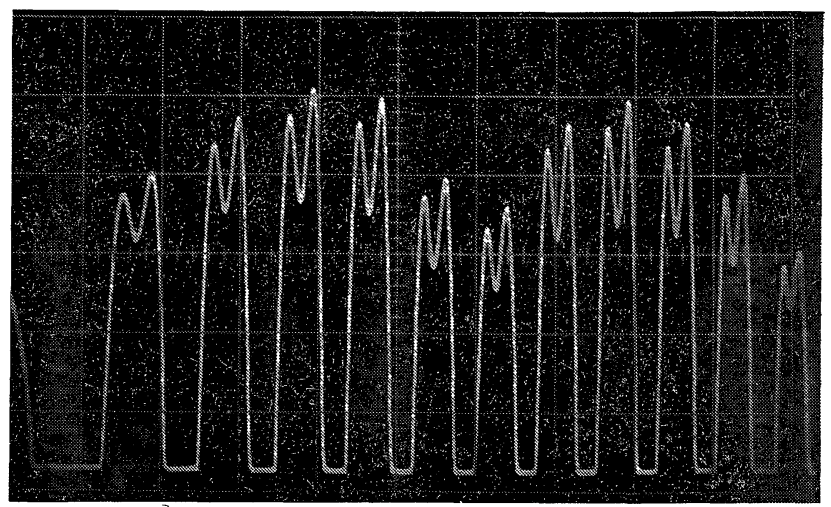

FIg. $3 a$. - Variation de $P_{2}$ en fonction de l'accord de la cavité $L_{2}$.

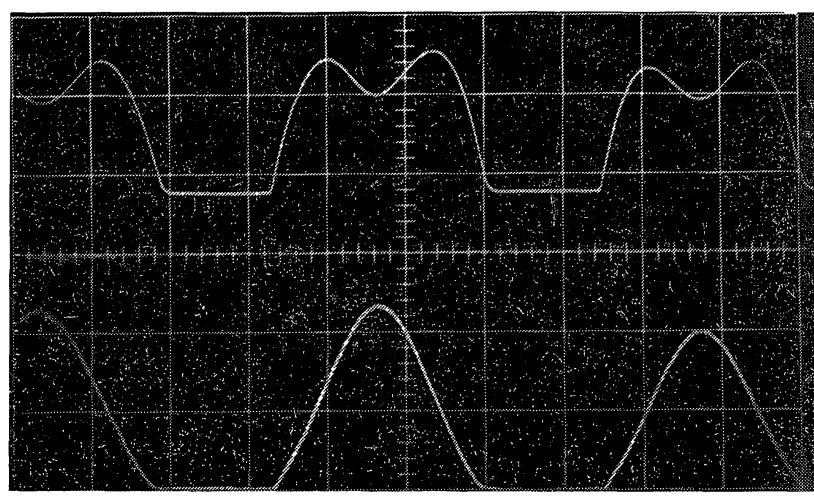

Fig. $3 b$. - Variations relatives de $P_{2}$ et $P_{3}$ en fonction de l'accord de la cavité $L_{2}$. Pour $P_{3}$ l'échelle a été multipliée par 150 (courbe du bas).

vibration linéaire en deux vibrations elliptiques dont les fréquences dépendent du champ. De plus un désaccord de la fréquence par rapport au centre de la raie provoque un dichroïsme circulaire qui conduit, pour les champs inférieurs à $H_{c}$, à une polarisation elliptique. Pour la raie $6328 \AA$ qui est une raie à gain faible, la rotation Faraday et le dichroïsme circulaire sont très faibles et font que dans un laser fortement anisotrope comme ici, la polarisation reste pratiquement linéaire. La rotation est négligeable et le vecteur lumineux reste dans le plan d'incidence des lames de Brewster, pour toutes les valeurs possibles de $\mathbf{H}$, et pour toutes les valeurs du désaccord $\delta$ du mode par rapport au centre de la raie (Fig. 2). Ceci se vérifie expérimentalement pour le laser utilisé.

L'étude de l'intensité de sortie d'un laser anisotrope [5] a permis de mettre en évidence expérimentalement les résonances résultant des effets non linéaires prévus par la théorie self-consistante de Lamb étendue aux lasers Zeeman [7].

L'intensité des composantes circulaires $\sigma^{+}$et $\sigma^{-}$ sont décrites par les relations :

$$
\begin{aligned}
& \dot{I}_{-}=2 I_{-}\left(\alpha_{-}-\beta_{-} I_{-}-\theta_{-+} I_{+}\right) \\
& \dot{I}_{+}=2 I_{+}\left(\alpha_{+}-\beta_{+} I_{+}-\theta_{+-} I_{-}\right)
\end{aligned}
$$

où les coefficients $\alpha$ représentent les gains non saturés 
des composantes, $\beta$ leur saturation propre, et $\theta$ leur couplage. Une courbe de l'intensité en fonction du champ magnétique, pour un laser du type Fox-Smith .est représentée sur la figure 4. Elle est caractéristique

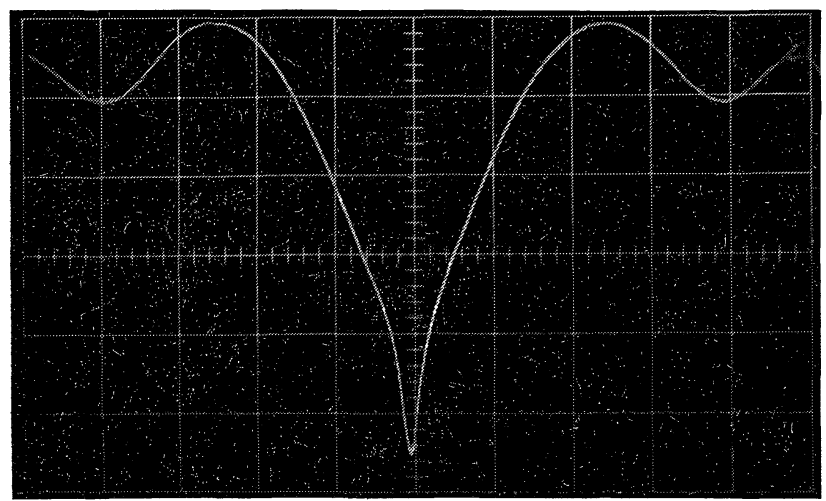

FIG. 4. - Intensité de sortie du laser en fonction du champ magnétique quand $H$ varie de $-H$ à $+H(1$ carreau correspond à $40 \mathrm{G}) \delta_{0} \simeq 280 \mathrm{MHz}$.

d'une valeur donnée du désaccord $\delta_{0}$ du mode. On constate que les effets non linéaires étudiés pour un laser monomode court [5], sont ici très marqués. La résonance centrale en champ nul correspond au coefficient $\theta$ et le Lamb dip magnétique intervenant pour une valeur $H_{0}$ du champ correspond au coefficient $\beta$ de saturation propre.

Le Lamb dip magnétique est caractéristique d'une composante $\sigma$ de la raie, c'est-à-dire soit de $\beta_{-}$, soit de $\beta_{+}$selon le signe $\delta_{0}$. Il est décalé de la fréquence centrale de la raie par effet Zeeman. Pour un désaccord $\delta_{0}$ donné, on obtient le Lamb dip magnétique d'une composante $\sigma$ pour un champ $H_{0}$, tel que le déplacement Zeeman $\Delta v_{z}$ de la composante soit :

$$
\Delta v_{z}=\delta_{0} \text {. }
$$

Pour fixer la fréquence, soit encore $\delta_{0}$, on appliquera un champ constant $H_{0}$ satisfaisant l'éq. (2) et on cherchera à se maintenir au creux du Lamb dip magnétique [8].

III. Principe et réalisation du système de stabilisation. - La fréquence du laser, caractéristique de la cavité active est stabilisée autour de la valeur $\delta_{0}$ correctement choisie. (Ici $\delta_{0} \simeq 280 \mathrm{MHz}$, il est nécessaire que le Lamb dip magnétique soit nettement marqué, donc bien séparé de la résonance en champ nul.) $\mathrm{Au}$ champ constant $H_{0}$ correspondant (la relation (2) donne $H_{0} \simeq 150$ gauss), est superposée une petite composante alternative $H_{1} \cos \omega t$ qui provoque une légère modulation de l'intensité à la fréquence $\omega$ et la phase de cette modulation changera de signe lorsque $\delta-\delta_{0}$ changera de signe. Nous avons donc ainsi un discriminant qui permet de stabiliser $\delta$ autour de $\delta_{0}$. Ceci est réalisé par la boucle 1 de la figure 1 . Le signal passe par un détecteur de phase, dont la tension continue de sortie est amplifiée puis appliquée à la céramique piézo-électrique portant le miroir $M_{3}$ pour corriger la longueur de la cavité $L_{1}$ et stabiliser la fréquence.

L'intensité du laser va dépendre de la cavité passive. Les courbes de la figure 3 nous montrent que pour stabiliser le laser sur un des maxima de la puissance utile $P_{2}$, il suffit de détecter la puissance $P_{3}$ et de la comparer à une tension de référence. La boucle 2 , pour une tension de référence donnée, corrigera les variations résultant des dérives de la cavité passive. La constante de temps de cette boucle qui doit corriger des dérives plus lentes, devra être plus grande. De cette façon, les corrections de déplacement de fréquence dû aux variations de puissance seront corrigées systématiquement par la boucle 1 .

Pour éviter, toute oscillation de la raie infrarouge $3,39 \mu$, une cuye à méthane est disposée dans la cavité. L'interféromètre de Fox-Smith (*) est constitué par un bloc d'invar pour limiter les dérives. Une plaque de numétal permet d'éviter tout effet magnétostrictif pouvant résulter de l'application de la composante alternative $H_{1}$ 。

IV. Résultats. - Nous avons réalisé des enregistrements de l'intensité de sortie du laser successivement, sans boucle de stabilisation, puis avec la boucle 1 seule, et enfin avec les deux boucles. Les courbes de la figure 5 montrent les résultats obtenus. La présence de la seconde boucle permet d'obtenir un système stable sur des durées beaucoup plus longues.
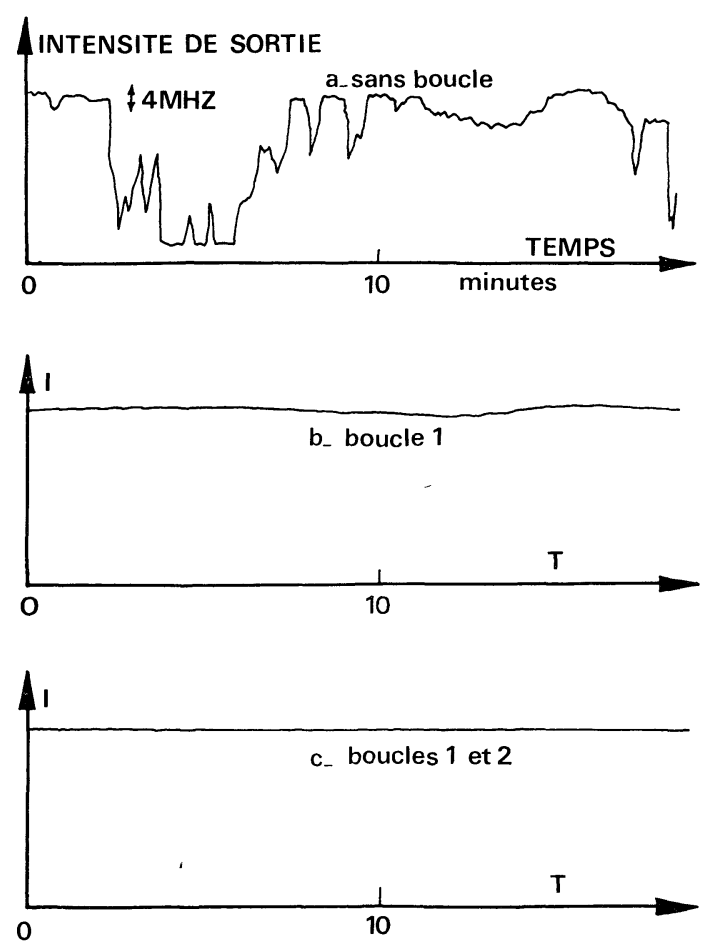

FIG. 5. - Enregistrements de l'intensité de sortie en fonction du temps.

(*) Nous remercions le groupe laser du laboratoire de spectroscopie herztienne de l'ENS pour le prêt de l'interféromètre qu'il a conçu et réalisé. 
En ce qui concerne la stabilité en fréquence les premières mesures faites précédemment sur un laser ordinaire [8] ont donné par la méthode des battements de photons, utilisant un laser Spectra-Physics modèle 119, une stabilité inférieure à $\pm 0,5 \mathrm{MHz}$. Ici nous avons

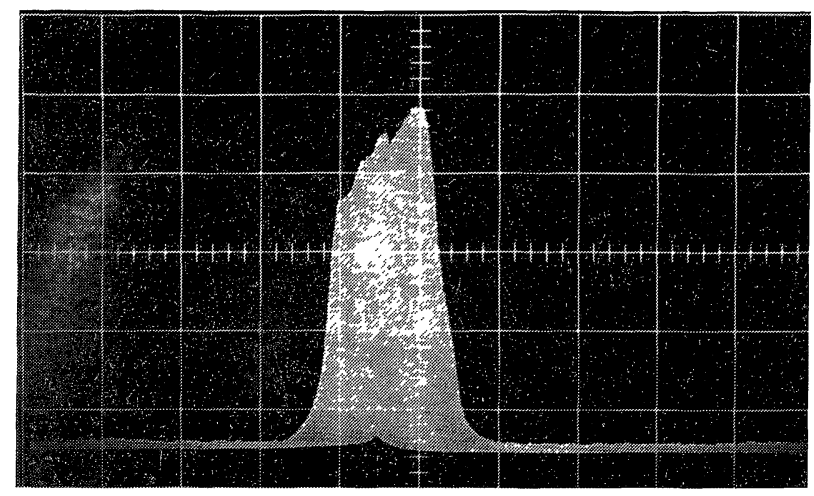

FIG. 6a. - Sans boucles de stabilisation (temps de pose $10 \mathrm{mn}$ ).

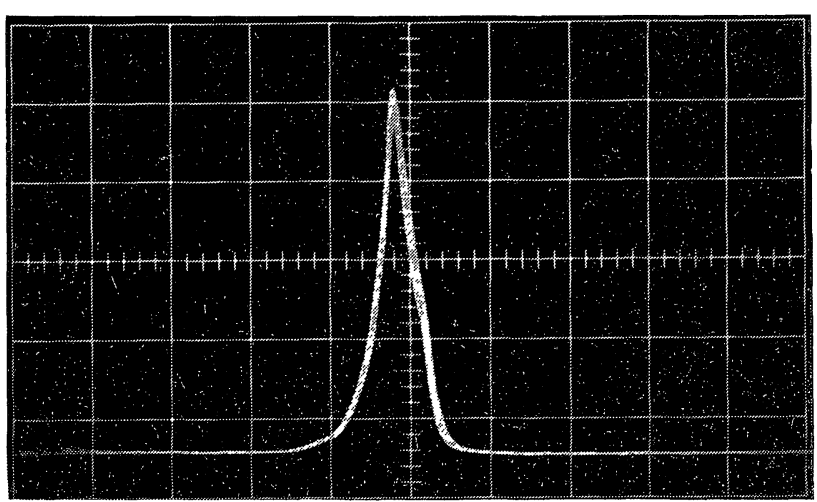

FIG. $6 b$. - Avec les deux boucles (temps de pose $10 \mathrm{mn}$ ).

FIG. 6. - Analyse au Pérot-Fabry de la fréquence du laser ; échelle $30 \mathrm{MHz}$ par division. seulement analysé la fréquence de sortie avec un PérotFabry (Tropel modèle 420 ) dont le pouvoir de résolution est d'environ $7 \mathrm{MHz}$, sur une durée de l'ordre de $10 \mathrm{mn}$ (Fig. 6). Les figures 5 et 6 permettent d'évaluer la stabilité en fréquence à environ $\pm 0,5 \mathrm{MHz}$.

On peut noter que le fait de faire varier la tension de référence de la boucle 2 , permet de régler la puissance de sortie du laser de façon continûment variable, le point de fonctionnement se déplaçant sur la courbe $P_{3}$. L'utilisation du Lamb dip magnétique permet d'éviter la modulation de fréquence supplémentaire superposée (d'environ $5 \mathrm{MHz}$ ), qui existe dans tout système utilisant le Lamb dip classique (inapplicable à ce montage). Ici le mode reste fixe et le profil Doppler est déplacé par effet Zeeman. De plus l'amplitude choisie pour $H_{1}$, qui peut être choisie relativement grande, permet d'avoir un meilleur rapport signal/bruit. La stabilité en fréquence peut être nettement supérieure à celle obtenue par le Lamb dip classique. La fréquence stabilisée est accordable dans un domaine de quelques centaines de Mégahertz, grâce au champ continu $H_{0}$. La stabilisation de la seconde cavité peut se faire directement à partir de la courbe $P_{2}$ en prélevant une faible partie du signal. Il suffit de se placer en un point de la courbe où la pente n'est pas nulle et de comparer à une tension de référence. Toute fluctuation de la puissance utile $P_{2}$ est alors directement corrigée.

Conclusion. - Nous avons proposé une nouvelle méthode de stabilisation d'un laser monomode de puissance qui utilise une référence atomique basée sur le Lamb dip magnétique. Une première boucle de contre-réaction stabilise la fréquence du laser et une seconde l'intensité de sortie. Pour tester les possibilités ultimes de la méthode il serait nécessaire de réaliser deux exemplaires identiques et d'étudier leur battement.

Bibliographie

[1] Voir par exemple :

Greytak (T.) and Benedek (G.), Phys. Rev. Letters, 1966, 17, 179 ; Proceedings of the IEEE, october $1965,1623$.

Durand (G. E.) and PINe (A. S.), IEEE J. Quantum Electronics, 1968, QE 4, 523.

[2] Sмiтн (S. W.), IEEE J. of Quantum Electronics, 1965, QE 1, 343.

Damaschini (R.), C. R. Acad. Sci., Paris, 1969, 268, 1169.

Liberman (S.), Thèse, Paris, 1971, 44.

[3] Sмith (P. W.), IEEE J. of Quantum Electronics, 1966, QE 2, 666.

[4] Cho (Y.), Tajime (Т.), Matsuo (Y.), IEEE J. of Quantum Electronics, 1968, QE 4, 669; 1970 sixth intern. Quantum Electronics conference.
[5] Le Floch (A.), Brun (P.), C. R. Acad. Sci., Paris, 1969, 269, 23.

[6] Le Floch (A.) and Le Naour (R.), Phys. Rev., 1971, A 4, 290 et les références citées dans cet article.

Le Floch (A.) et Stephan (G.), C. R. Acad. Sci., Paris, 1972, 274, 491.

Le Floch (A.) et Stephan (G.), à paraître, Phys. Rev. A., August, 1972.

[7] SARgent (M.), III, LAMB (W. E.) jr, and Fork (R. L.), Phys. Rev., 1967, 164, 450.

DURAND (G.), IEEE J. of Quantum electronics, 1966, 2, 448 ; Ann. Institut H. Poincaré, 1966, 4, 263.

[8] Le Floch (A.), Frere (P.) and Brun (P.), Appl. Phys. Letters, 1970, 17, 40 ; Brevet Anvar n 6943193. 